\title{
A critical threshold for global pediatric surgical workforce density
}

\author{
Megan E. Bouchard ${ }^{1}$ (D) $\cdot$ Yao Tian ${ }^{1} \cdot$ Jeanine Justiniano ${ }^{2} \cdot$ Samuel Linton $^{1} \cdot$ Christopher DeBoer $^{1} \cdot$ Fizan Abdullah $^{1}$. \\ Monica Langer ${ }^{1}$
}

Accepted: 31 May 2021 / Published online: 9 June 2021

(c) The Author(s), under exclusive licence to Springer-Verlag GmbH Germany, part of Springer Nature 2021

\begin{abstract}
Purpose 1.7 billion children lack access to surgical care, particularly in low- and middle-income countries (LMIC). The pediatric surgical workforce density (PSWD), an indicator of surgical access, correlates with survival of complex pediatric surgical problems. To determine if PSWD also correlates with population-level health outcomes for children, we compared PSWD with pediatric-specific mortality rates and determined the PSWD associated with improved survival.

Methods Using medical licensing registries, pediatric surgeons practicing in 26 countries between 2015 and 2019 were identified. Countries' PSWD was calculated as the ratio of pediatric surgeons per 100,000 children. The correlation between neonatal, infant and under 5 mortality rates and PSWD was assessed using Spearman's correlations and piecewise linear regression models.

Results Four LIC, eight L-MIC, ten UMIC and four HIC countries, containing 420 million children, were analyzed. The median PSWD by income group was 0.03 (LIC), 0.12 (L-MIC), 1.34 (UMIC) and 2.13 (HIC). PSWD strongly correlated with neonatal $(0.78, p<0.001)$, infant $(0.82, p<0.001)$ and under $5(0.83, p<0.001)$ mortality rates. Survival improved with increasing PSWD to a threshold of 0.37 .

Conclusion PSWD correlates with pediatric population mortality rates, with significant improvements in survival with PSWD $>0.37$. Currently, PSWD in LMICs is inadequate to meet UN Sustainable Development Goal 3.2 for child mortality.
\end{abstract}

Keywords Workforce density $\cdot$ Pediatric surgery $\cdot$ Childhood mortality $\cdot$ National surgical planning

\section{Introduction}

Despite major improvements in childhood survival in lowand middle-income countries (LMICs), the 2015 Millennium Developmental Goals for neonatal, infant, and child mortality were not met, thus prompting inclusion in the United Nation's Sustainable Developmental Goal (SDG) 3.2: to end preventable deaths of children under 5 years of age by 2030 [1]. Surgery is now unanimously recognized as an essential component of universal health coverage by the 2015 World Health Assembly; access to pediatric

Megan E. Bouchard

mbouchard@luriechildrens.org

1 Division of Pediatric Surgery, Department of Surgery, Northwestern University Feinberg School of Medicine, Ann \& Robert H. Lurie Children's Hospital of Chicago, 225 E. Chicago Ave, Chicago, IL 60611, USA

2 Loyola University Chicago Stritch School of Medicine, 2160 S. First Ave, Maywood, IL 60153, USA surgical services, specifically, is critical to effectively care for congenital conditions and trauma, which disproportionately affect children in LMICs and contribute to preventable morbidity and mortality [2,3]. With nearly one-fifth of surgical disability-adjusted life-years (DALYs) caused by congenital or perinatal conditions and the known lack of surgical access in LMICs, access to pediatric surgical care remains paramount [4]. However, an estimated 1.7 billion children worldwide lack access to surgical care, including 1.1 billion in low- and middle-income countries (LMICs) where children comprise nearly $50 \%$ of the population $[5,6]$.

Among other indicators of surgical access, the Lancet Commission on Global Surgery (LCoGS) identified the density of surgical providers as a marker of sufficient workforce capacity [7]. The LCoGS demonstrated a strong correlation between surgical workforce density and adult population health outcomes, specifically maternal mortality rate, with a critical threshold of 20 surgical providers per 100,000 people correlating with significant reductions in mortality [7]. This threshold has served as a benchmark 
for surgical access and a target for national surgical planning for scale-up of the surgical workforce in LMICs. While it is well recognized that specific pediatric surgery workforce goals are needed for national strategic planning to improve pediatric care, none currently exist $[5,8]$.

Specialist pediatric surgeons are needed for treatment of congenital and complex pediatric problems, thus the pediatric surgical workforce density (PSWD) is an important indicator of access to comprehensive pediatric care [9]. Outcomes for specific pediatric surgical conditions in LMICs correlate with PSWD as evidenced by a recent systematic review demonstrating that a PSWD of 0.4 per 100,000 children significantly correlated with increased odds of survival in gastroschisis, esophageal atresia, intestinal atresia, and typhoid perforation [9].

While it makes sense that outcomes from surgical conditions correlate with PSWD, it is unknown if PSWD correlates with pediatric-specific population mortality rates. Therefore, we aimed (1) to assess for a correlation between PSWD and neonatal, infant, and under 5 childhood mortality rates and (2) to identify the critical PSWD threshold associated with significant mortality reduction for national strategic surgical planning.

\section{Methods}

\section{Study design and data sources}

We conducted a retrospective, cross-sectional study of pediatric surgeons' workforce density (PSWD) using publicly available medical licensing registries and the WHO Global Surgical Workforce Database [10]. Only countries with publicly available data on the subspecialty classification of physicians were included. A convenience sample of 26 geographically and economically diverse countries between 2015 and 2018 was identified.

Countries included in the analysis were classified by the World Bank income brackets including low income (LIC), lower-middle income (L-MIC), upper-middle income (UMIC) and high income (HIC). For each country, the most recent publicly available medical licensing data were used to identify the number of practicing pediatric surgeons with an active license. Pediatric surgeons were defined by country-specific certification as specialist pediatric surgeons.

Given most countries define pediatric care as care delivered to patients less than 15 years old, each country's population less than 15 years old was retrieved from the World Bank [6, 9]. Additionally, the under 5-year old, infant and neonatal mortality rates for each country were obtained [6].

\section{Outcomes and analysis}

The primary outcome was each country's respective PSWD, calculated as the number of pediatric surgeons per 100,000 children less than 15 years old. Then for each income bracket, the median PSWD and mean proportion of less than 15-year-old population were determined. The under 5 , infant, and neonatal mortality rates were converted to survival rates ( 1 -mortality rate) [9]. Next, the logarithmic under 5 , infant and neonatal survival rates were plotted as a function of PSWD [7]. Spearman's correlation coefficients were then used to assess the strength of correlation for each survival rate, using an alpha level of 0.05 to determine significance. Finally, following the LCoGS's methodology, piecewise linear regression models were used to determine the critical PSWD threshold, or inflection point, at which there was a significant increase in survival [7].

\section{Results}

A convenience sample of 26 countries was identified: four LIC, eight L-MIC, ten UMIC and four HIC countries, comprising 420,764,643 children (Table 1). The mean proportion of the population less than 15 years old decreased with rise in World Bank income bracket, with $42.8 \%$ for LICs, $38.2 \%$ for L-MICs, $21.6 \%$ for UMICs, and $18.7 \%$ for HICs. In LIC countries, the PSWD ranged from 0.02 to 0.24 , median 0.03 . For L-MICs, the PSWD ranged from 0.03 to 0.36 , median 0.12. For UMICs, the PSWD ranged from 0.27 to 7.14 , median 1.34. Finally, in HICs, the PSWD ranged from 1.15 to 3.60 , median 2.13 .

\section{PSWD correlation with pediatric-specific mortality rates}

For under 5-years old, infant, and neonatal mortality rates, there was an observed positive correlation between survival rates and PSWD. Countries with higher PSWD demonstrated improved survival rates for each age group (Fig. 1). Furthermore, each survival rate strongly correlated with PSWD: under 5 -years old $(0.83, p<0.001)$, infant $(0.82$, $p<0.001)$ and neonatal $(0.78, p<0.003)$ (Table 2$)$.

A critical threshold of 0.37 PSWD correlated with significant increases in survival, beyond which gains were observed, but the gradient of the curve was flatter (Fig. 1, Table 2). All four LICs, all eight L-MICs and one UMIC were below the critical threshold of 0.37 PSWD. The LIC and L-MICs also reported a higher proportion of their populations aged less than 15 years old $(42.8 \%$ and $38.2 \%$, respectively). Combined, the number of children in countries 


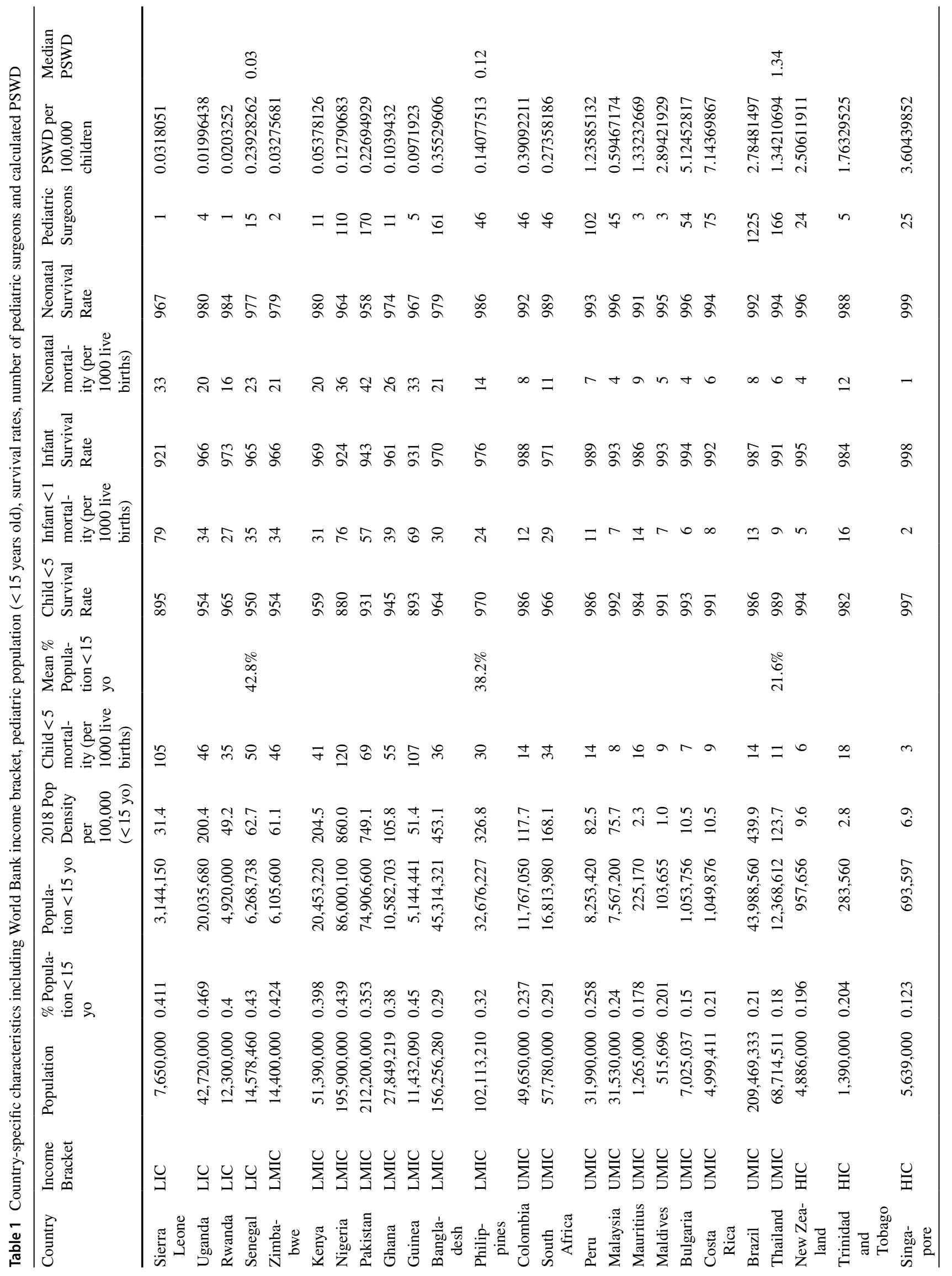




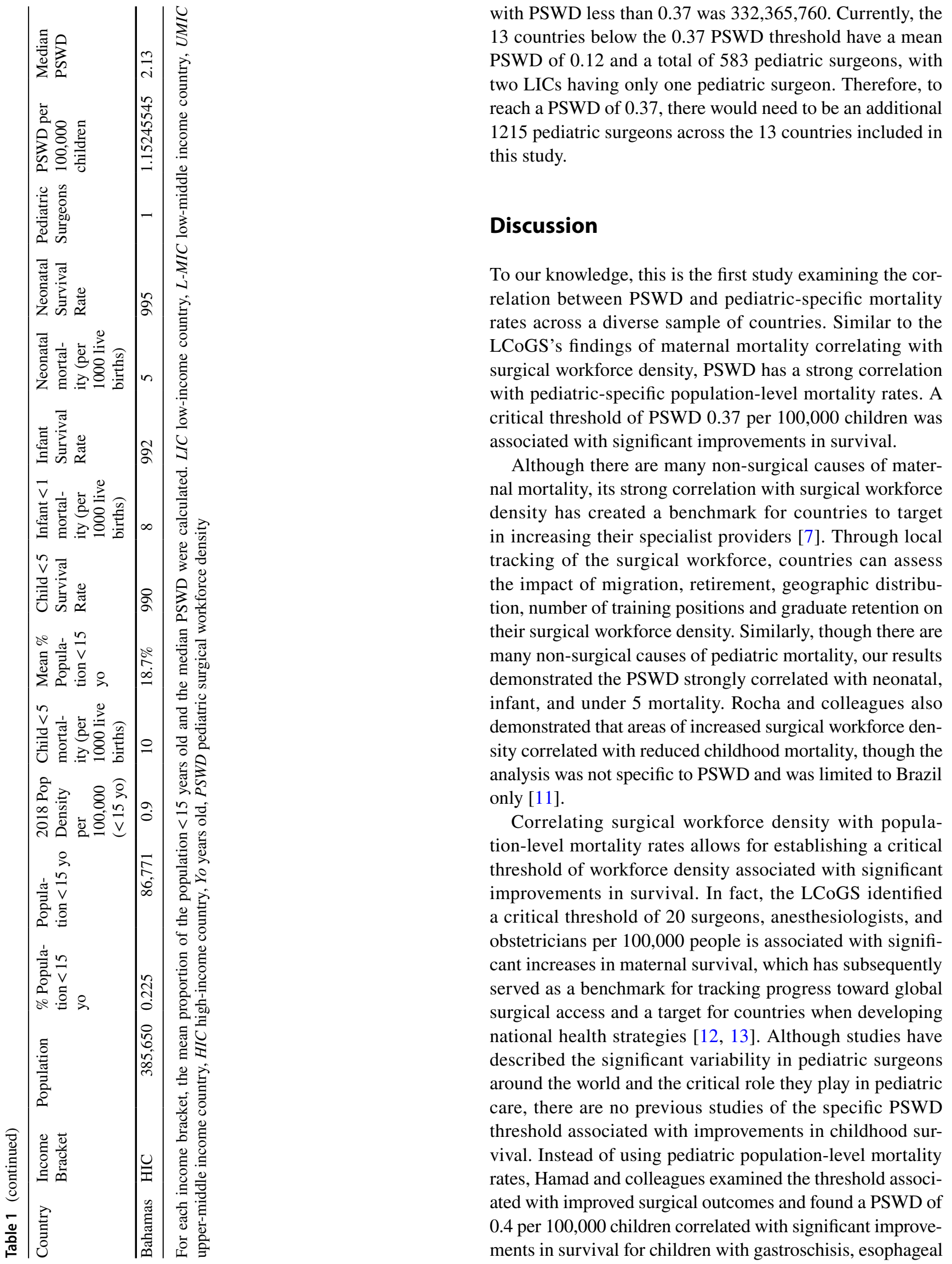



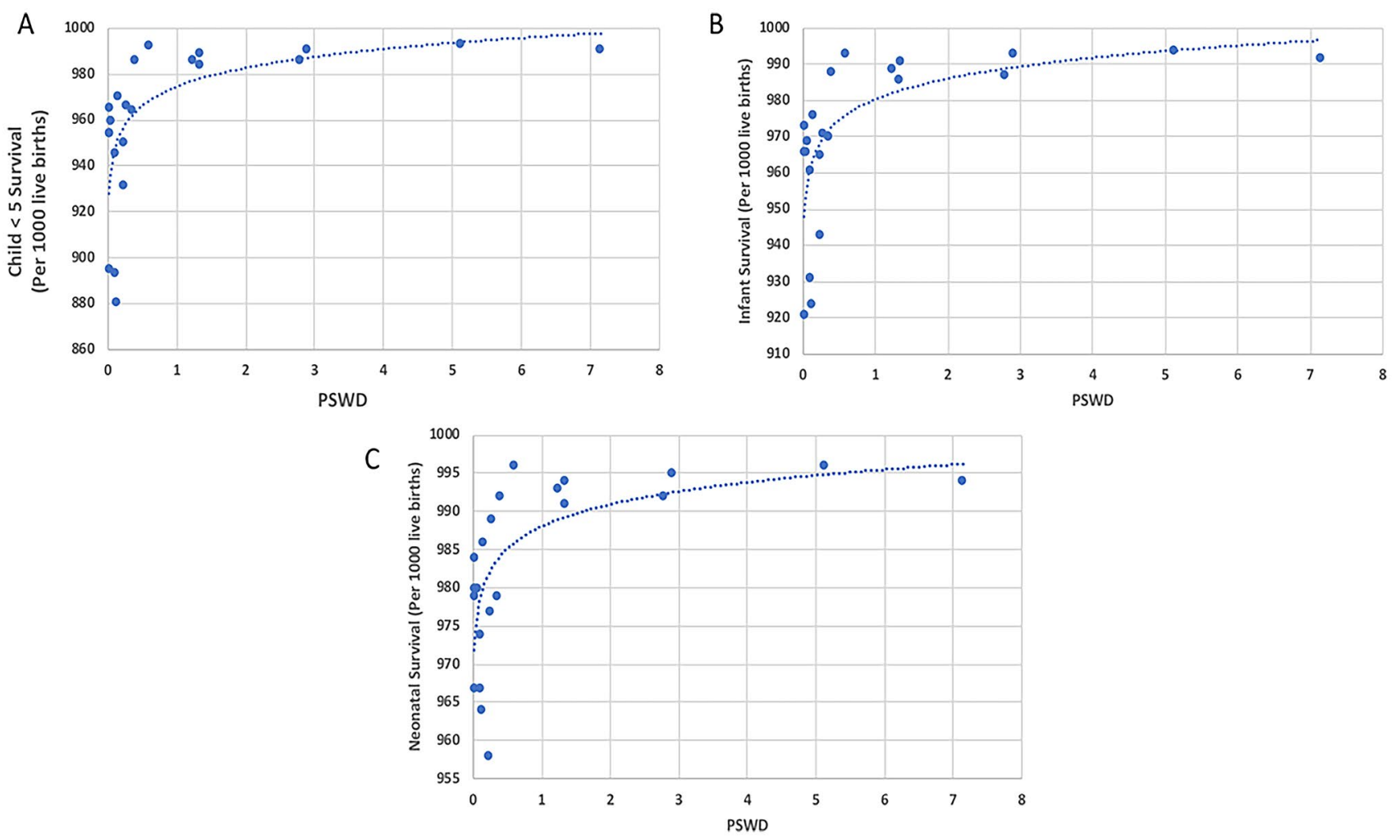

Fig. 1 Child $<5$ years old (A), infant (B) and neonatal (C) survival rates plotted as a function of PSWD. PSWD pediatric surgical workforce density

Table 2 Spearman's calculation demonstrating the strength of correlation between neonatal, infant and under 5-year-old survival rates with PSWD

\begin{tabular}{llll}
\hline & Neonatal & Infant & Child $<5$ \\
\hline Spearman correlation coefficient & $0.78(p<0.001)$ & $0.82(p<0.001)$ & $0.83(p<0.001)$ \\
$\begin{array}{l}\text { Critical PSWD threshold (per 100,000 } \\
\text { children) }\end{array}$ & 0.37 & 0.37 & 0.37 \\
\hline
\end{tabular}

The critical PSWD threshold that correlated with significant gains in survival for each mortality curve is also displayed atresia, intestinal atresia and typhoid perforation [9]. The authors argued this PSWD of 0.4 established a novel benchmark for the scale-up of the pediatric surgical workforce [9]. In comparing PSWD with pediatric population-level mortality rates, we demonstrated a PSWD of 0.37 correlated with significant improvements in childhood survival. Therefore, a PSWD of $0.37-0.4$ per 100,000 children is associated with not only improved outcomes for surgical conditions, but also increased pediatric survival across the population [9]. This PSWD threshold could serve as a goal/metric in national surgical, obstetric and anesthetic planning (NSOAP), where a diverse group of medical and government stakeholders work to identify a country's urgent health needs and strategically allocate resources accordingly $[7,8]$.

Unfortunately, all LMICs (and one UMIC) sampled were below the critical threshold PSWD of 0.37 , representing 332 million children with inadequate access to surgical care. This PSWD deficit is especially critical in LMICs, where a greater proportion of the population is less than 15 years old and there are higher birth rates, with a greater prevalence of complex, congenital conditions [13, 14]. Thus, many children will continue to die from treatable conditions if this deficit is not addressed. Additionally, pediatric patients often require specialized anesthesia care; thus, its availability may be a rate-limiting step to providing surgical care, even with available surgical providers [15]. Currently, efforts are underway to increase the PSWD through targeted training programs such as the collaboration between the College of Surgeons of East, Central, and South Africa (COSECSA), the West African College of Surgeons (WACS), and KidsOR - a charitable organization working to build surgical infrastructure in LMICs $[16,17]$. These programs aim to 
support the development of 120 centers of excellence in pediatric surgical care across Africa, including training pediatric surgeons, anesthesiologists, nurses, and surgical technicians and providing high quality, pediatric-specific operating theatres [18].

Additional strategies have included task shifting to other surgeons, physicians or surgical technicians, when appropriate $[15,17]$. A common practice in most countries, general surgeons frequently provide non-complex pediatric surgical care with equivalent outcomes; and while they are not a substitute for pediatric surgeons for complex and congenital conditions, they provide critical and timely surgical care, especially in rural regions [19]. The Global Initiative for Children's Surgery outlined the optimal resources, infrastructure and personnel needed to provide pediatric surgical care specific to each condition $[16,18]$. Initiatives to provide training for local providers at district hospitals, who are often the first point of contact for children with surgical problems, have been successfully implemented around the globe. For example, trauma training for non-specialist providers in low-resource settings in sub-Saharan Africa demonstrated significant improvements in knowledge and clinical confidence [16]. Meanwhile, in India, successful training and support initiatives were implemented to instruct rural providers on basic perioperative pediatric orthopedic, general surgery and anesthesia care [20]. Many LMICs have also demonstrated success in increasing surgical volume and access through training surgical technicians to independently perform basic surgical procedures, maintaining high-quality outcomes with faster and less costly training than specialist surgeons, though no studies exist on whether this is applicable to pediatric surgical conditions $[9,21]$. While this strategy does not eliminate the need for pediatric surgeons for complex and congenital conditions, it may increase surgical accessibility for children with basic surgical needs and provide relief to an overburdened and limited pediatric surgical workforce. Whether through these existing efforts or emerging initiatives, there is an urgent need to increase the pediatric surgical workforce to meet the United Nations' SDG 3.2, to significantly reduce childhood mortality $[5,8]$.

While this study offers a novel assessment of the correlation between PSWD and pediatric population-level mortality rates, there are some notable limitations. First, the sample was limited to 26 countries that provide publicly available and subspecialty-specific licensing data. Although the sample was geographically and economically diverse, this sample may not be representative of the PSWD in other countries with similar resources. Second, in some countries, we were unable to determine if providers practice year-round in country or are retired; however, the majority of countries provide annual information on whether the license is active, suggesting the number of retired surgeons inadvertently included was very low. Third, many other providers besides pediatric surgeons provide surgical care to children, including general surgeons, other specialist surgeons (neurosurgeons, orthopedic surgeons, urologists, plastic surgeons, etc.) and other physicians or surgical technicians. Using licensing data alone, one cannot determine the number of non-surgical physicians or other providers who deliver surgical care in a given country. While these providers do provide important care, our focus was on pediatric surgeons specifically, as they are critical to providing comprehensive care for complex and congenital conditions. Finally, this study was limited to a retrospective analysis; a prospective study would be more useful to clearly identify the number of currently practicing pediatric surgeons in each country and the impact of unforeseen circumstances, such as the added stress from canceled surgeries during the COVID-19 pandemic to already overburdened health systems in LMICs.

Future analyses should increase the sample size of countries analyzed and prospectively assess the impact of interventions to increase the PSWD in LMICs. Additionally, research is needed to assess the correlation of pediatric population-level mortality rates with pediatric anesthesia workforce density, as they are critical to providing successful surgical care for children. Finally, it will be important to prospectively assess the impact of increased PSWD in LMICs on surgical volume, surgical outcomes and overall childhood survival. PSWD is only one indicator of surgical access and is almost certainly an indirect representation of other resources, infrastructure, and specialists needed for comprehensive pediatric care such as intensive care, anesthesiology, nursing and advanced nutritional support. Nevertheless, pediatric surgeons remain an essential component of a functional health system and PSWD can serve as a target for national health planning.

\section{Conclusion}

PSWD correlates with population mortality rates for neonates, infants and children under 5 years' old, with a critical threshold of 0.37 PSWD associated with a significant increase in survival. These novel findings suggest the current PSWD in LMICs, where a greater proportion of the population is less than 15 years old, have a higher birth rate, and have a greater prevalence of complex and congenital surgical conditions, is inadequate to meet the UN SDG 3.2 for infant and under 5 mortality. To improve the overall survival of children globally, further efforts are needed to increase the training and retention of pediatric surgeons in LMICs and to expand the associated health workforce, infrastructure, and services needed to care for infants and children with surgical conditions. 
Funding No funding source was secured for this study.

\section{Declarations}

Conflict of interest The authors have no conflicts of interest relevant to this work to disclose.

Ethical approval The authors all agree they have complied with the journal's ethical policies.

\section{References}

1. Sustainable Development Goal 3 (2020) United Nations. https:// unstats. un. org $/ \mathrm{sdgs} / \mathrm{metadata} ? \mathrm{Text}=\& \mathrm{Goal}=3 \&$ Target $=3.2$. Accessed Nov 17, 2020

2. Price R, Makasa E, Hollands M (2015) World Health Assembly resolution WHA68.15: "Strengthening emergency and essential surgical care and anesthesia as a component of universal health coverage"-addressing the public health gaps arising from lack of safe, affordable and accessible surgical and anesthetic services. World J Surg 39:2115-2125

3. Sitkin NA, Ozgediz D, Donkor P et al (2015) Congenital anomalies in low- and middle-income countries: the unborn child of global surgery. World J Surg 39(1):36-40. https://doi.org/10.1007/ s00268-014-2714-9

4. Ologunde R, Maruthappu M, Shanmugarajah K et al (2014) Surgical care in low and middle-income countries: burden and barriers. Int J Surg 12(8):858-863

5. Mullapudi B, Grabski D, Ameh E et al (2019) Estimates of number of children and adolescents without access to surgical care. Bull World Health Organ 97(4):254-258

6. World Bank Open Data (2020) World Bank. https://data.world bank.org/. Accessed Sep 9, 2020

7. Meara JG, Leather AJ, Hagander L et al (2015) Global Surgery 2030: evidence and solutions for achieving health, welfare, and economic development. Lancet 386(9993):569-624. https://doi. org/10.1016/S0140-6736(15)60160-X

8. Wasserman I, Peters AW, Roa L et al (2020) Breaking specialty silos: improving global child health through essential surgical care. Glob Health Sci Pract 8(2):183-189. https://doi.org/10.9745/ GHSP-D-20-00009

9. Hamad D, Yousef Y, Caminsky NG et al (2020) Defining the critical pediatric surgical workforce density for improving surgical outcomes: a global study. J Pediatr Surg 55(3):493-512. https:// doi.org/10.1016/j.jpedsurg.2019.11.001
10. Global Surgical Workforce Database (2017) World Health Organization. https://www.who.int/gho/health_workforce/surgical/surge ons/en/. Accessed Sep 9, 2020

11. Rocha TAH, Vissoci J, Rocha N et al (2020) Towards defining the surgical workforce for children: a geospatial analysis in Brazil. BMJ Open 10(3):e034253. https://doi.org/10.1136/bmjop en-2019-034253

12. Guest GD, McLeod E, Perry WRG et al (2017) Collecting data for global surgical indicators: a collaborative approach in the Pacific Region. BMJ Glob Health 2:e000376. https://doi.org/10.1136/ bmjgh-2017-000376

13. Bouchard ME, Justiniano J, Vervoort D, et al. (2020) Cross-sectional analysis tracking workforce density in surgery, anesthesia, and obstetrics as an indicator of progress toward improved global surgical access. Int J Surg: Global Health 3(6)

14. Lalchandani P, Dunn JC (2015) Global comparison of pediatric surgery workforce and training. J Pediatr Surg 50(7):1180-1183. https://doi.org/10.1016/j.jpedsurg.2014.11.032

15. Optimal Resources for Children's Surgical Care: Version 2.0 (2019). Global Initiative for Children's Surgery

16. Peter NA, Pandit H, Le G et al (2016) Delivering a sustainable trauma management training programme tailored for low-resource settings in East, Central and Southern African countries using a cascading course model. Injury 47(5):1128-1134. https://doi.org/ 10.1016/j.injury.2015.11.042

17. Goodman L, St-Louis E, Yousef Y et al (2018) The global initiative for children's surgery: optimal resources for improving care. Eur J Pediatr Surg 28(01):51-59

18. What We Do (2020) KidsOR: Surgery for Children. https://www. kidsor.org/what-we-do/training/. Accessed October 30, 2020

19. Judhan RJ, Silhy R, Statler K et al (2015) The integration of adult acute care surgeons into pediatric surgical care models supplements the workforce without compromising quality of care. Am Surg 81(9):854-858

20. Vellore: Royal College of Surgeons Children's Training Partnership (2019) Global Initiative for Children's Surgery. https://www. globalchildrenssurgery.org/2019/02/12/vellore-royal-college-ofsurgeons-project/. Accessed October 30, 2020

21. Falk R, Taylor R, Kornelsen J et al (2020) Surgical task-sharing to non-specialist physicians in low-resource settings globally: a systematic review of the literature. World J Surg 44:1368-1386

Publisher's Note Springer Nature remains neutral with regard to jurisdictional claims in published maps and institutional affiliations. 